\title{
EDITORIAL \\ An ethical framework for deep brain stimulation in children
}

\author{
Benjamin Davidson, MD, ${ }^{1}$ Lior M. Elkaim, ${ }^{2}$ Nir Lipsman, MD, PhD, ${ }^{1,3}$ and \\ George M. Ibrahim, MD, PhD ${ }^{1,4}$
}

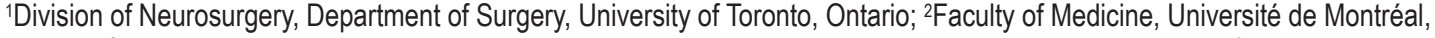
Québec; ${ }^{3}$ Division of Neurosurgery, Sunnybrook Health Sciences Centre, University of Toronto, Ontario; and ${ }^{4}$ Division of Neurosurgery, Hospital for Sick Children, Program in Neuroscience and Mental Health, Hospital for Sick Children Research Institute, University of Toronto, Ontario, Canada
\end{abstract}

$\mathrm{D}$ EEP brain stimulation (DBS) is increasingly considered for the treatment of refractory neurodevelopmental conditions affecting children. ${ }^{25}$ In 1999, Coubes and colleagues were among the first to report on the use of pediatric DBS for primary dystonia. ${ }^{9}$ Since then, pediatric DBS has gained considerable traction, with several case series reported. ${ }^{25,29}$ Although dystonia remains the most common indication for pediatric DBS, the technology has also been applied to treat epilepsy ${ }^{44}$ and Tourette syndrome..$^{38}$ More recently, DBS of the nucleus accumbens and amygdala have been reported for self-injurious behaviors. ${ }^{34,41}$

The majority of the evidence for DBS in children has been derived from the adult literature, in which the procedure has been shown to be safe and effective for an increasing number of indications. ${ }^{37}$ However, pediatric DBS has many substantial differences, with important bioethical, social, and legal considerations. First, patients and their caregivers represent a uniquely vulnerable population. Second, given biological differences between adults and children, the procedure in pediatric populations can be considered a surgical innovation and is still investigational for all indications. Third, the natural history of a disease within the context of a child's neuromuscular and cognitive development must be factored into treatment decisions. Furthermore, the surgical risks and technical nuances of DBS differ from those in adults. Thus, evidence from the adult DBS literature may not be readily translatable to pediatric populations.

With the exception of DYTI dystonia (for which there is level II-2 evidence ${ }^{45}$ ), all indications for DBS in pediatric patients are in the early stages of investigation; thus, the procedure represents a surgical innovation, i.e., a procedure that "departs in a significant way from standard or accepted practice." 11 Surgical innovations are charac- terized by evolving techniques, outcome measures, and patient selection. ${ }^{31}$ Three systematic reviews and metaanalyses (two manuscripts currently under review and one paper in $\operatorname{press}^{10}$ ) have described the conduct of DBS in pediatric populations (Table 1), all emphasizing significant heterogeneity and the need for prospective studies to guide patient selection. The framework we describe here is intended to be applied to DBS in children in all settings, most of which are presently experimental or under the realm of surgical innovation. Ultimately, the most basic ethical principles protecting research subjects flow from the Nuremberg Code (1948), the Declaration of Helsinki (1964), and the Belmont Report (1979); however, in children with a medically complex condition who may benefit from novel investigational treatments, a more targeted framework is needed..$^{11,14,43}$

Herein we expand on differences in the conduct of DBS between adults and children and the unique ethical challenges they pose. First, we describe ethical issues raised by the vulnerability of the patient population, the novelty of the application of DBS for expanding indications in children, and the conduct of the procedure in children despite limited evidence. We then propose a bioethical framework for the evaluation of children for DBS. Although ethical issues may figure more or less prominently depending on the individual patient and the indication, the framework we put forth may be generalized to shape personal, institutional, and social policies regarding DBS in children. It is not our intention to prescribe the steps and thought process for carrying out pediatric DBS, but rather to advance dialogue regarding the ethical underpinnings of this promising technique in children.

The Population: Ethical Challenges in Vulnerable Patients

The pediatric population being considered for DBS is 
TABLE 1. Systematic reviews and meta-analyses of DBS in exclusively pediatric populations

\begin{tabular}{ccc}
\hline Authors \& Year & \multicolumn{1}{c}{ Indication } & $\begin{array}{c}\text { No. of Subjects } \\
\text { (total no. of studies) }\end{array}$ \\
\hline $\begin{array}{c}\text { Elkaim et al., } \\
2018^{*}\end{array}$ & $\begin{array}{c}\text { Primary \& secondary } \\
\text { dystonia }\end{array}$ & $\begin{array}{c}\text { Primary: 173; secondary: } \\
\text { 139; myoclonus: } 9(72)\end{array}$ \\
\hline $\begin{array}{c}\text { Coulombe et al., } \\
2018 \text { (in press) }\end{array}$ & Tourette's syndrome & $58(21)$ \\
\hline Yan et al., 2018* & Epilepsy & $40(21)$ \\
\hline
\end{tabular}

* Manuscripts currently under review.

complex and vulnerable, requiring a high level of casespecific ethical appraisal. Ethical criteria for DBS in vulnerable adult populations have been published: ${ }^{24} 1$ ) adequate discussion of patient selection, 2) provision of an evidence-based rationale that exceeds a standard of reasonableness, 3) demonstration of the disease burden, 4) transparency in informed consent, 5) multidisciplinary review of individual cases, 6) adequate oversight of all aspects of the research, and 7) routine scheduled evaluations focused on ethical aspects of the trial design. ${ }^{24}$ While many of these principles apply to the conduct of DBS in children, several unique pediatric considerations should be recognized.

Children may not be able to articulate their wishes due to intellectual immaturity or neurocognitive disability, leaving caregivers to make difficult decisions. In cases in which the child has not yet declared their interests, decisions are based on the child's presumed best interest rather than their right to autonomy and self-determination.,20 Such decision-making spans not only the perioperative period, but also subsequent decision points such as device programming, further treatment, and potential revisions..$^{20}$ Depending on the child's age and abilities at initial intervention, he or she may begin assuming a greater role in subsequent decision-making, an evolution that all stakeholders within the circle of care must anticipate.

There is no accepted age at which children are deemed competent and capable of medical decision-making. Competence is more often associated with prior experience rather than a specific age. ${ }^{2,20}$ Beginning at age 7 years, children with a prior surgical history are often asked to participate in decision-making. ${ }^{1}$ In the case of DBS, there is usually no prior experience, and few such cases have been performed; therefore, advice and experience from family support groups with related experience are limited. Children with prior non-DBS surgical experience might participate better in consent discussions regarding general surgical risks, but would be unlikely to have added insight into the specifics of undergoing DBS.

In some cases, there is a dilemma between early versus delayed intervention to allow the child to better participate in decision-making. A delay in treatment may result in undue harm from disease sequelae, such as musculoskeletal deformity from spastic conditions, ${ }^{6,28,29}$ worsening epilepsy, and so forth. Early intervention is most warranted for conditions with strong evidence such as primary DYT1 dystonia, but is less indicated where evidence is hetero- geneous such as in secondary dystonia. ${ }^{25}$ The concept of "double jeopardy" may apply: First, these children suffer as a result of their disabilities, and second, lower priority is given to treatments that may improve their quality of life. ${ }^{18}$ A "relational" view of decision-making holds that treatment (and evidence for and against intervention) should be considered within the context of the child's subjective experience with the illness. ${ }^{12,20,39}$ Consultation with medical bioethicists may be prudent to maintain the strictest standards for patient care.

\section{The Procedure: Known and Unknown Risks of Surgical Innovation}

Although DBS for movement disorders in adults is an accepted treatment option, in children it still represents a surgical innovation, even for the most common indication, dystonia. As mentioned above, an innovation is defined as a procedure that significantly departs from standard or accepted practice and is characterized by evolving techniques, outcome measures, and patient selection. ${ }^{11,31}$ The extent to which a surgical innovation deviates from standard practice is directly related to the extent to which it requires ethical oversight and regulation. ${ }^{5}$

Before proposing DBS for a pediatric patient, the health team should recognize that the risks, and often the benefits, of the procedure are not well understood. Available data are limited to small case series with specific institutional protocols that are poorly generalizable to other centers. Risks of the procedure may be higher in children when that procedure is performed using protocols derived from adult DBS. For example, early case series on pediatric DBS have reported higher rates of infection ranging from 5\% to $33 \%$, as compared to approximately $5 \%$ with adult DBS. ${ }^{22,29,35}$ Since then, two series in which the pulse generator was implanted at a later date both reported an infection rate of $0 \% .{ }^{21,33}$ Furthermore, DBS leads and batteries are sized for use in adults, and the risks of skin erosion and hardware fracture seem to be higher in children. ${ }^{25,29}$ An appreciation of the ways in which a standard procedure in adults (i.e., DBS) may be an innovative intervention in children is critical to determining the extent of ethical oversight required for its use in clinical and experimental indications.

Surgical innovations are also associated with evolving outcome measures..$^{31}$ Current instruments used to measure the success of DBS treatment may not be ideal, even for the most widely studied indication, dystonia. Originally created to assess primary torsion dystonia in adults, the Burke-Fahn-Marsden Dystonia Rating Scale (BFMDRS) has been adopted for pediatric use and is now the most commonly reported measure of dystonia impairment. ${ }^{7}$ Intended for use in secondary dystonia, the Barry-Albright Dystonia Scale (BADS) is also frequently cited. ${ }^{4}$ These scales often fail to capture a child's experience with illness (i.e., dystonia). For example, a recent study highlighting this showed that DBS improved individualized functional goals in the absence of significant changes in BFMDRS scores in children with secondary dystonia. ${ }^{16}$

Despite the uncertainties introduced by evolving techniques, patient selection, and outcome measures, clinicians have an obligation to apply DBS in appropriately selected 
candidates. Future studies will aim to clarify the extent to which pediatric DBS deviates from the adult equivalent, as well as the minimum level of evidence required before acceptance of the procedure. ${ }^{19}$

\section{The Element of Time: Interaction Between Disease Course and the Child's Development}

Timing plays a much larger role in pediatric DBS. Unlike in adults, when the decision to proceed with DBS involves ensuring that the disease is truly refractory to other treatments, the pediatric neurosurgeon must also factor in the child's development. The most common indication for pediatric DBS is dystonia, for which early intervention seems to yield better motor improvements. ${ }^{25,30}$ In treating secondary dystonia due to cerebral palsy, those treated before skeletal maturity improved in their BFMDRS scores by $38 \%$, while those treated after skeletal maturity improved by only $9 \% .{ }^{30}$ Early interventions for dystonia likely mitigate contractures and may prevent or inhibit fixed deformities from developing. ${ }^{21,30}$ Advantages of early intervention must be counterbalanced by the various challenges associated with DBS in a younger child.

Ethical challenges are also presented by diseases with an unknown natural history. In Tourette syndrome, a tic disorder whose treatment has shown promising results in adult DBS series, pediatric cases often spontaneously remit in adolescence, making early DBS less appealing. ${ }^{23,25}$ While most guidelines recommend against DBS for Tourette syndrome in children for this reason, the result is that a subset of children must endure years of unnecessary suffering until their disease is deemed to be nonremitting. ${ }^{36,40}$ Moreover, anorexia nervosa, a common and severe condition often beginning during adolescence, is associated with a high rate of treatment resistance and death and may therefore be amenable to earlier DBS. ${ }^{26}$

\section{The Technical Nuances: Ethical Challenges Introduced by Technical Considerations}

Technical nuances in children may also present ethical challenges. One example of this is the need for general anesthesia in children, which may preclude microelectrode recordings, potentially decreasing the accuracy of lead placement. In adults, lead misplacement is the chief cause of suboptimal outcomes with DBS. ${ }^{13}$ There is reasonable case-control evidence from adults with Parkinson's disease that awake and asleep DBS yield similar results. ${ }^{8}$ This comparison has not yet been performed in children. Awake DBS has been successfully performed in many children over the age of 10 , although it requires innovative strategies such as an intraoperative child-life specialist and preoperative relaxation training. ${ }^{29}$

In children under the age of 7 , the location of DBS electrodes can also change with head growth. The deepest lead should be placed slightly ventral to the target in children with further head growth anticipated. ${ }^{27}$ Similarly, extra wire should be loosely looped in the subcutaneous pocket to avoid lead fracture with growth.

Stimulation parameters also introduce an element of uncertainty in children, for whom no standardized protocols exist. ${ }^{29}$ In adults, common stimulation settings have been demonstrated to be safe through autopsies on patients who had chronic DBS. ${ }^{29}$ Safety data of this nature are not available in children. Consent discussions for pediatric DBS should outline the limitations of our current state of knowledge, especially as regards the safety profile and long-term effects of stimulation. An ethical obligation of the scientific community is to facilitate the standardization of pediatric DBS protocols, including programming.

The ethical challenges associated with these procedural differences highlight the importance of appreciating and communicating that pediatric DBS is novel not only because of the age group, but also because the basic technical procedure is still being refined.

\section{The Evidence: Translating From Adult to Pediatric Populations}

Although children are biologically different from adults, the evidence used to guide pediatric DBS is largely derived from adult data. Stimulation parameters for dystonia DBS were adopted from the adult Parkinson's disease experience, with most patients receiving $>100 \mathrm{~Hz}$ of stimulation. ${ }^{3}$ In a relatively large case series, Alterman et al. demonstrated that dystonia may respond well to much lower-frequency stimulation, i.e., $60 \mathrm{~Hz} .{ }^{3}$ Stimulation frequencies and voltages have been demonstrated to be safe in adults, causing only mild gliosis, but such reports are not available in children. ${ }^{29}$ Despite a few prior successful treatments, clinicians should be aware and communicate to families that there is simply not a strong body of evidence regarding the use of these devices in children, yet.

Because the evidence for pediatric DBS remains in its infancy, the neurosurgeon is often acting as a clinician and scientist concurrently. "Therapeutic misconception" occurs when study participants fail to recognize the potentially competing roles and obligations of the treating physician as both clinician and scientist. ${ }^{32}$ Patient populations that hold particularly negative views about their health are at higher risk for therapeutic misconception. ${ }^{17}$

Various strategies can be implemented to circumvent knowledge gaps and mitigate the risk of therapeutic misconception. The Fort Worth pediatrics program in Texas uses institutional board review to ensure that patients and families are adequately informed and fairly selected. ${ }^{29}$ The program also uses an independent nurse to perform a parallel consent process. Regardless of the institutionspecific process, each case should be carefully considered by a multidisciplinary group, factoring in whatever degree of input the child is capable of providing along with the decision of a competent substitute decision maker.

\section{Ethical Framework for Pediatric DBS}

Ethical frameworks function as scaffolding for public, institutional, and personal views toward an existing problem. ${ }^{15}$ Ethical frameworks include common themes that transcend individual challenges, such as access, accountability, autonomy, client centeredness, collaboration, efficiency, equity, evidence, prevention, public involvement, quality, responsibility, sustainability, and protection of the vulnerable. ${ }^{15}$ Given the ethical challenges unique to the conduct of DBS in children, we propose a bioethical 


\section{TABLE 2. Guidelines for the ethical conduct of DBS in children}

Research design \& conduct in the child's best interest
Hypotheses, outcomes, \& procedures viewed in a developmentally
relevant context
Realistic discussion of what is known \& what is unknown regarding the
risk profile in pediatric DBS; weighing the decision to proceed with
\& the timing of DBS vs other treatments with better known efficacy
\& risk profiles
Cautious application of adult data to the design of pediatric clinical
trials
Diligently report methods \& results to the neurosurgical community in
the spirit of collaboration

framework centered around five primary expectations that may serve to advance dialogue regarding pediatric DBS (Table 2).

\section{Expectation 1: Protection of the Child's Best Interest}

Decisions and clinical hypotheses and their testing should be made in the spirit of the child's best interest. Since children are often unable to declare their treatment preferences because of immaturity or disability, treatments that support their development and decrease their suffering should be emphasized. An examination of a child's best interest may also encompass benefit to caregivers. ${ }^{42}$ For example, reduced dystonia may facilitate the delivery of care and hygiene to the child by their caregivers, thus providing a net benefit. Although DBS research, for instance, in the form of clinical trials, is primarily designed to test an intervention that may benefit future patients, surgical trials are inherently accompanied by a reasonable expectation of benefit to the individual subject. Efforts to uphold the highest standards of ethical principles and mitigate therapeutic misconception during the conduct of clinical trials involving DBS in children are paramount.

\section{Expectation 2: Decisions Made Within the Developmental Context}

The use of DBS in children should be viewed through a developmental lens. The treating team should have a clear concept of the expected trajectory of the child's illness, their development, and how the proposed treatment fits within this larger picture. The timing of any proposed treatment should be clearly justified based on these factors, such as trying to prevent contractures from developing in the case of dystonia. For example, in refractory Tourette syndrome, the benefits of intervention should be weighed against an unknown and potentially favorable natural history. A holistic approach encompassing the child's medical, psychiatric, and social comorbidities and position should inform the presurgical discussions.

\section{Expectation 3: Mitigating "Relative" Known and Unknown Risks to the Individual Child}

Relatively little is known about the risks of DBS in pediatric patients and how they differ from those in adults, and all members of the treating team should be aware of this. In the conduct of pediatric DBS, the child's rela- tive experience with illness must be weighed against uncertainty to minimize known and theoretical risks. The child's subjective experience should dictate the degree to which DBS can and should be pursued for an accepted or investigational indication.

The known benefits and harms of a child's current treatment, as well as their subjective experience of illness, should be balanced against the relatively unknown risks and benefits of DBS. For example, intrathecal baclofen therapy is an established, effective treatment for secondary generalized dystonia. The point at which DBS should be considered represents a balance of its unknown risks (and benefits) relative to the efficacy and tolerance of the established treatment (intrathecal baclofen). Presently, in most cases, the balance tips toward DBS when other treatments have become ineffective or harmful despite significant suffering due to the illness. As better data emerge, the decision to proceed with and the timing of the intervention is expected to be associated with less uncertainty and to pose fewer ethical dilemmas.

\section{Expectation 4: Avoidance of Overreliance on the Adult Literature}

The treating team and caregivers must be constantly cognizant that "children are not small adults." In addition to the unique risk and benefit profiles, the diseases manifested in children differ significantly from those in adults, as does a child's perception of illness. Incumbent on the treating physician is the need to approach pediatric DBS as uncharted territory and to take on the burden of demonstrating reasonable evidence for and against all elements of the treatment.

\section{Expectation 5: Communicate and Collaborate With Other Practitioners}

Each lesson learned in the pre-, intra-, and postoperative phase of DBS in the pediatric setting is extremely valuable and should be disseminated to the larger clinical community. Both positive and negative results require discussion and publication. For instance, given the heterogeneity of outcomes following secondary dystonia, it is incumbent upon the medical community to better define the ideal surgical candidates. A frank review of complications must also occur, with institutional oversight. Such practices have already improved outcomes following DBS in children. ${ }^{21,33}$ Although small case series still provide valuable data, emphasis should be placed on collaborative registries, with standardized, prospective outcome reporting. Collaboration to harmonize protocols between institutions will be necessary to produce larger, more generalizable case series.

\section{Conclusions}

Current evidence for the use of DBS in children is limited to case reports and case series. Early results for pediatric DBS have generated enthusiasm for the expansion of its indications. Herein we have outlined some of the key ethical issues in implementing DBS in the pediatric population, as well as an ethical framework to guide neurosurgeons when considering DBS for accepted or experimental indications.

https://thejns.org/doi/abs/10.3171/2018.7.FOCUS18219 


\section{References}

1. Alderson P: Children's consent to treatment. Abstract debate is unhelpful. BMJ 307:260-261, 1993

2. Alderson P, Sutcliffe K, Curtis K: Children's competence to consent to medical treatment. Hastings Cent Rep 36:25-34, 2006

3. Alterman RL, Miravite J, Weisz D, Shils JL, Bressman SB, Tagliati M: Sixty hertz pallidal deep brain stimulation for primary torsion dystonia. Neurology 69:681-688, 2007

4. Barry MJ, VanSwearingen JM, Albright AL: Reliability and responsiveness of the Barry-Albright dystonia scale. Dev Med Child Neurol 41:404-411, 1999

5. Bernstein M, Bampoe J: Surgical innovation or surgical evolution: an ethical and practical guide to handling novel neurosurgical procedures. J Neurosurg 100:2-7, 2004

6. Blumetti FC, Wu JC, Barzi F, Axt MW, Waugh MC, Selber P: Orthopaedic surgery in dystonic cerebral palsy. J Pediatr Orthop [epub ahead of print], 2016

7. Burke RE, Fahn S, Marsden CD, Bressman SB, Moskowitz C, Friedman J: Validity and reliability of a rating scale for the primary torsion dystonias. Neurology 35:73-77, 1985

8. Chen T, Mirzadeh Z, Chapple KM, Lambert M, Shill HA, Moguel-Cobos G, et al: Clinical outcomes following awake and asleep deep brain stimulation for Parkinson disease. J Neurosurg [epub ahead of print March 16, 2018; DOI: 10.3171/2017.8.JNS17883]

9. Coubes P, Echenne B, Roubertie A, Vayssière N, Tuffery S, Humbertclaude V, et al: [Treatment of early-onset generalized dystonia by chronic bilateral stimulation of the internal globus pallidus. Apropos of a case.] Neurochirurgie 45:139-144, 1999 (Fr)

10. Coulombe ME, Alotaibi NM, Gorman DA, Kalia S, Lipsman N, Lozano A, et al: Deep brain stimulation for Gilles de la Tourette syndrome in children and youth: a meta-analysis with individual participant data. J Neurosurg Pediatr [in press], 2018

11. Department of Health, Education, and Welfare: Protection of human subjects; Belmont Report: notice of report for public comment. Fed Regist 44:23191-23197, 1979

12. Donchin A: Understanding autonomy relationally: toward a reconfiguration of bioethical principles. J Med Philos 26:365-386, 2001

13. Ellis TM, Foote KD, Fernandez HH, Sudhyadhom A, Rodriguez RL, Zeilman P, et al: Reoperation for suboptimal outcomes after deep brain stimulation surgery. Neurosurgery 63:754-761, 2008

14. Gandevia B, Tovell A: Declaration of Helsinki. Med J Aust 2:320-321, 1964

15. Giacomini M, Kenny N, DeJean D: Ethics frameworks in Canadian health policies: foundation, scaffolding, or window dressing? Health Policy 89:58-71, 2009

16. Gimeno H, Tustin K, Lumsden D, Ashkan K, Selway R, Lin JP: Evaluation of functional goal outcomes using the Canadian Occupational Performance Measure (COPM) following deep brain stimulation (DBS) in childhood dystonia. Eur $\mathbf{J}$ Paediatr Neurol 18:308-316, 2014

17. Goebel S, von Harscher M, Mehdorn HM: Comorbid mental disorders and psychosocial distress in patients with brain tumours and their spouses in the early treatment phase. Support Care Cancer 19:1797-1805, 2011

18. Harris J: Life: quality, value and justice. Health Policy 10:259-266, 1988

19. Ibrahim GM, Fallah A, Snead OC III, Drake JM, Rutka JT, Bernstein M: The use of high frequency oscillations to guide neocortical resections in children with medically-intractable epilepsy: how do we ethically apply surgical innovations to patient care? Seizure 21:743-747, 2012

20. Ibrahim GM, Fallah A, Snead OC III, Elliott I, Drake JM,
Bernstein M, et al: Ethical issues in surgical decision making concerning children with medically intractable epilepsy. Epilepsy Behav 22:154-157, 2011

21. Johans SJ, Swong KN, Hofler RC, Anderson DE: A stepwise approach: decreasing infection in deep brain stimulation for childhood dystonic cerebral palsy. J Child Neurol 32:871875, 2017

22. Kenney C, Simpson R, Hunter C, Ondo W, Almaguer M, Davidson A, et al: Short-term and long-term safety of deep brain stimulation in the treatment of movement disorders. J Neurosurg 106:621-625, 2007

23. Leckman JF, Peterson BS, Pauls DL, Cohen DJ: Tic disorders. Psychiatr Clin North Am 20:839-861, 1997

24. Lipsman N, Bernstein M, Lozano AM: Criteria for the ethical conduct of psychiatric neurosurgery clinical trials. Neurosurg Focus 29(2):E9, 2010

25. Lipsman N, Ellis M, Lozano AM: Current and future indications for deep brain stimulation in pediatric populations. Neurosurg Focus 29(2):E2, 2010

26. Lipsman N, Lam E, Volpini M, Sutandar K, Twose R, Giacobbe P, et al: Deep brain stimulation of the subcallosal cingulate for treatment-refractory anorexia nervosa: 1 year follow-up of an open-label trial. Lancet Psychiatry 4:285294, 2017

27. Lumsden DE, Ashmore J, Charles-Edwards G, Selway R, Lin JP, Ashkan K: Observation and modeling of deep brain stimulation electrode depth in the pallidal target of the developing brain. World Neurosurg 83:438-446, 2015

28. Lumsden DE, Gimeno H, Elze M, Tustin K, Kaminska M, Lin JP: Progression to musculoskeletal deformity in childhood dystonia. Eur J Paediatr Neurol 20:339-345, 2016

29. Marks WA, Honeycutt J, Acosta F, Reed M: Deep brain stimulation for pediatric movement disorders. Semin Pediatr Neurol 16:90-98, 2009

30. Marks WA, Honeycutt J, Acosta F Jr, Reed M, Bailey L, Pomykal A, et al: Dystonia due to cerebral palsy responds to deep brain stimulation of the globus pallidus internus. Mov Disord 26:1748-1751, 2011

31. McKneally MF: The ethics of innovation: Columbus and others try something new. J Thorac Cardiovasc Surg 141:863866, 2011

32. Miller FG, Brody H: A critique of clinical equipoise. Therapeutic misconception in the ethics of clinical trials. Hastings Cent Rep 33:19-28, 2003

33. Olaya M, Alsina L, de Sevilla MF, Catalá A, López-Ramos MG, Martín Mateos MA, et al: Epstein-Barr virus infection triggering a haemophagocytic syndrome. Allergol Immunopathol (Madr) 42:627-629, 2014

34. Park HR, Kim IH, Kang H, Lee DS, Kim BN, Kim DG, et al: Nucleus accumbens deep brain stimulation for a patient with self-injurious behavior and autism spectrum disorder: functional and structural changes of the brain: report of a case and review of literature. Acta Neurochir (Wien) 159:137143,2017

35. Piacentino M, Pilleri M, Bartolomei L: Hardware-related infections after deep brain stimulation surgery: review of incidence, severity and management in 212 single-center procedures in the first year after implantation. Acta Neurochir (Wien) 153:2337-2341, 2011

36. Roessner V, Plessen KJ, Rothenberger A, Ludolph AG, Rizzo R, Skov L, et al: European clinical guidelines for Tourette syndrome and other tic disorders. Part II: pharmacological treatment. Eur Child Adolesc Psychiatry 20:173-196, 2011

37. Rumberg F, Bakir MS, Taylor WR, Haberl H, Sarpong A, Sharankou I, et al: The effects of selective dorsal rhizotomy on balance and symmetry of gait in children with cerebral palsy. PLoS One 11:e0152930, 2016 (Erratum in PLoS One 12:e0177585, 2017)

38. Servello D, Porta M, Sassi M, Brambilla A, Robertson MM: 
Deep brain stimulation in 18 patients with severe Gilles de la Tourette syndrome refractory to treatment: the surgery and stimulation. J Neurol Neurosurg Psychiatry 79:136-142, 2008

39. Sherwin S: A Relational Approach To Autonomy In Health-Care. Philadelphia: Temple University Press, 1988

40. Steeves T, McKinlay BD, Gorman D, Billinghurst L, Day L, Carroll A, et al: Canadian guidelines for the evidence-based treatment of tic disorders: behavioural therapy, deep brain stimulation, and transcranial magnetic stimulation. Can J Psychiatry 57:144-151, 2012

41. Sturm V, Fricke O, Bührle CP, Lenartz D, Maarouf M, Treuer $\mathrm{H}$, et al: DBS in the basolateral amygdala improves symptoms of autism and related self-injurious behavior: a case report and hypothesis on the pathogenesis of the disorder. Front Hum Neurosci 6:341, 2013

42. Taylor DC: Treating children and adults: whose body is it anyway? Eur Child Adolesc Psychiatry 8:315-319, 1999

43. United Nations: Convention on the Rights of the Child. Geneva: United Nations, 1989 (https://www.ohchr.org/EN/
ProfessionalInterest/Pages/CRC.aspx) [Accessed July 10, 2018]

44. Valentín A, Selway RP, Amarouche M, Mundil N, Ughratdar I, Ayoubian L, et al: Intracranial stimulation for children with epilepsy. Eur J Paediatr Neurol 21:223-231, 2017

45. Vidailhet M, Vercueil L, Houeto JL, Krystkowiak P, Benabid AL, Cornu P, et al: Bilateral deep-brain stimulation of the globus pallidus in primary generalized dystonia. N Engl J Med 352:459-467, 2005

\section{Disclosures}

The authors report no conflict of interest.

\section{Correspondence}

George M. Ibrahim: george.ibrahim@sickkids.ca.

\section{INCLUDE WHEN CITING}

DOI: 10.3171/2018.7.FOCUS18219. 